\title{
Routine Screening of Blood and Urine for Severe Anticonvulsant Reactions in Asymptomatic Patients Is of Doubtful Value
}

\begin{abstract}
On rare occasions anticonvulsant medication may cause severe or fatal toxic reactions. This position paper, approved by the Canadian Association for Child Neurology, examines the value of routine blood and urine screening to prevent catastrophic reactions in asymptomatic patients. The basic assumptions of such a screening program are faulty or unproven. Although not definitive, available studies of screening do not appear to support its value. It is recommended that routine blood and urine screening be discontinued in asymptomatic patients. Until such time as further research is able to identify patients at special risk, it is suggested that patients be carefully informed of the early symptoms of severe toxic reactions and report these immediately to a physician.
\end{abstract}

RÉSUMÉ: La surveillance courante de sang et d'urine à propos de réactions graves aux médicaments antiépileptiques chez les patients asymptomatiques a valeur douteuse Les médicaments anti-épileptiques entraînent rarement des réactions sévères ou mortelles. Ce document-ci, approuvé par l'Association Canadienne de Neurologie Pédiatrique, constate la valeur de la surveillance courante de sang et d'urine afin d'éviter des réactions catastrophiques chez les patients asymptomatiques. Les présomptions fondamentales d'une telle surveillance sont erronnées ou manquent de la preuve. Quoi qu'elles ne soient pas encore définitives, les études disponibles de la surveillance ne semblent pas appuyer sa valeur. On propose que l'échantillonage habituel de sang et d'urine se discontinue chez les patients asymptomatiques. Jusque l'investigation peut identifier les patients à haute risque, on suggère d'informer aux patients en ce qui concerne les symptôms de réactions toxiques graves et on demande que les patients rapportent ces telles réactions tout de suite à leur médecin.

Can. J. Neurol. Sci. 1989; 16: 361-364

\section{Editors Note:}

This paper was originally published in the Canadian Medical Association Journal (1989; 140: 1303-1305). It is based on a report from an ad hoc committee of The Canadian Association for Child Neurology and was approved at the annual meeting of the Association in June 1988. Since there are still widely varying opinions amongst both pediatric and adult neurologists regarding the order of routine screening for anticonvulsant toxicity, we have invited some additional commentary which follows the report. The Journal would welcome further correspondence on this topic.

In 1965 DeVries recommended that patients receiving anticonvulsants be regularly screened with blood and urine tests to avoid rare catastrophic drug reactions. ${ }^{1}$ This recommendation has not been critically appraised and yet appears frequently for most anticonvulsants in the Compendium of Pharmaceuticals and Specialties (CPS) in Canada ${ }^{2}$ or the Physicians' Desk Reference (PDR) in the U.S.A. ${ }^{3}$ It is the premise of this paper that such routine screening is unlikely to be of help and, on occasion, may interfere with the treatment of the patient's epilepsy. We suggest that educating the patient or parent to promptly report early symptoms of severe anticonvulsant reactions should replace routine blood and urine screening in asymptomatic patients taking antiepileptic medications.

\section{BACKGROUND}

Anticonvulsants, on rare occasions, may induce a severe or fatal reaction including such conditions as aplastic anemia, hepatitis, nephritis and Stevens-Johnson Syndrome. ${ }^{4}$ The exact frequency of such reactions is uncertain but overall has been estimated to occur about $1 / 50,000$ patients prescribed an anticonvulsant. ${ }^{4}$ Screening to avoid such reactions involves three assumptions. First, it is assumed that these reactions are idiosyncratic and unpredictable in an individual patient. The work of Spielberg and coworkers suggests that eventually those at risk might be identifiable prior to treatment. ${ }^{5}$ They have demonstrated that some patients with severe adverse reactions to certain anticonvulsants have a genetically determined abnor- 
mality in arene oxide metabolism. Abnormalities in arene oxide have been demonstrated in the lymphocytes of patients with phenytoin associated hepatitis and phenytoin and carbamazepine associated aplastic anemia. 5.6 Despite this promising research, there does not yet appear to be a readily available way to mark a patient as significantly at risk for an adverse reaction.

Second, it is assumed that there is a presymptomatic phase before the reaction that can be detected by blood and urine screening. We are not aware that this assumption has been proven for any anticonvulsant drug. On the contrary, most reactions appear to have sudden onset. For example, a child died from valproate liver toxicity 1 week after a normal screening SGOT. ${ }^{7}$ Third, it is assumed that if a reaction is detected in its presymptomatic phase then discontinuing the drug would limit the severity of the reaction. Although possibly correct, this assumption has not been tested. In some cases it appears that once underway the reaction cannot be altered. 8

For these reasons, we submit that there is no clear medical rationale for the screening recommended in the CPS or PDR.

\section{Studies Of Screening For Anticonvulsant Reactions}

Two studies have directly addressed the screening question. In the first, 199 children with epilepsy were prospectively screened for liver, hematological and renal toxicity with conventional blood and urine testing before medication, 1 month, 3 months, 6 months after starting medication and then every 6 months. ${ }^{9}$ Drugs used included phenobarbital, phenytoin, carbamazepine and valproic acid. No serious clinical toxic events were detected and the number of abnormalities in each test was as great before medication as at any time on medication. Six percent of the tests had to be repeated because of abnormalities and yet all repeat tests showed results closer to normal even though there had been no change in the patients' medication doses. The authors noted that two children had their medication unnecessarily stopped as the result of screening abnormalities.

In the second study, 662 adults with newly diagnosed epilepsy were randomly assigned to carbamazepine, phenytoin, phenobarbital or primidone and screened prospectively over at least 6 months. ${ }^{10}$ None of the 622 patients stopped taking their medication because of drug-related changes in laboratory results. The authors concluded that "routine monitoring of hematology and serum chemistry batteries probably is not cost effective and of doubtful clinical value for healthy patients taking a single antiepileptic drug."

In neither study were there any patients with catastrophic reactions and, therefore, the issue of the sensitivity of blood and urine screening for these reactions has not been settled. A direct test of the value of screening is virtually impossible. The issue could only be completely settled by a randomized trial of screening vs no screening. We estimate that such a study would require a sample of about 1 million patients with epilepsy. ${ }^{9}$ The distinction between abnormal laboratory tests and severe anticonvulsant reactions is important. For example, in one study of 200 children receiving carbamazepine, 27 had a WBC of less than $4000 / \mathrm{cu} . \mathrm{ml}$ at some point in their treatment but none had clinical illness and the leukopenia was transient in nearly all.11 In 3, the neutropenia persisted (time not stated) and the drug was stopped, even though there were no clinical symptoms. In a detailed study of liver function tests in patients receiving valproic acid it was noted that $4 / 25$ patients developed increases in serum SGOT. ${ }^{2}$ In 3 of the 4 , the dose was reduced and the SGOT returned to normal. The other patient was symptomatic with leg edema and malaise, her valproic acid was stopped and she recovered. Thus, the specificity of an abnormal screening test is very low for a clinically significant toxic reaction.

\section{Problems With Screening}

Regular screening poses several problems. First, the frequency of screening recommended by the manufacturers is almost impossible to manage in the clinical setting, especially in the treatment of "needle shy" children. For example, the section on carbamazepine in the PDR recommends screening of blood counts, reticulocytes and serum iron pretreatment and weekly for 3 months and monthly thereafter for 2-3 years plus baseline and periodic evaluation of liver function. ${ }^{13}$ For valproic acid, the recommendations are slightly less specific but suggest liver function tests "at frequent intervals" especially during the first 6 months of treatment. ${ }^{14}$ We doubt that such stringent regimes are often clinically feasible.

Second, a basic premise in screening is that all screening test abnormalities, however slight, must be promptly rechecked and the patient re-examined immediately since any abnormality may mark the beginning of a severe reaction. In one study, quoted above, this meant repeating $6 \%$ of all tests. This is both time consuming and anxiety producing for family and physician. 14

Third, we suspect that some patients have successful antiepileptic treatment interrupted unnecessarily when abnormalities are observed in screening tests.

Lastly, the screening process is very expensive. If every patient with epilepsy in North America had a CBC and SGOT three times per year, the total annual cost would be in excess of $\$ 400,000,000$. This amount is far in excess of all research money devoted annually in North America to the understanding of epilepsy. ${ }^{9}$ This figure does not include physician time or time lost from work by families.

\section{Alternatives To Blood And Urine Screening}

If we assume that routine blood and urine screening is ineffective, bothersome to patient care and expensive, there needs to be another way to approach the very real but rare problem of severe anticonvulsant reactions. Patients need to be told of the remote danger of these catastrophic reactions despite their rarity. They should not be led to a false sense of security by a screening program. Instead, they should be informed of the symptoms that occur early in a toxic reaction and report these immediately. A rash is the first warning for the most frequent severe reaction to phenobarbital, carbamazepine, and phenytoin. ${ }^{4}$ Liver failure associated with valproic acid is almost always heralded by anorexia, lethargy and vomiting. ${ }^{15}$ Aplastic anemia associated with any anticonvulsant is accompanied by infection, bruising and symptoms of anemia. We recognize that the value of such a "clinical screening" program remains to be determined but we believe that it, at least, is a more realistic attempt to identify patients with serious drug reactions as early as possible in the reaction. 
More research needs to be devoted to prediction of who will develop toxic reactions. Studies such as those noting the association of valproate toxicity with young age (especially under two years) and polytherapy may reduce the number of patients with valproate liver failure by limiting the use of the drug in this higher risk group. 15 Caution in using phenytoin in patients undergoing radiotherapy may decrease some of the severe exfoliative dermatitis problems induced by phenytoin. ${ }^{8}$ It is probable that some patients will continue to suffer severe adverse effects from anticonvulsants regardless of the number of contraindications that are proven. However, routine blood and urine screening does not appear at all likely to prevent these disasters.

\title{
RECOMMENDATIONS
}

1. Before starting an anticonvulsant patients should be informed, preferably in writing, of the possible severe adverse reactions and the early symptoms of the reaction. They should be warned to contact their physician immediately if these symptoms develop.

2. Prior to treatment, baseline liver function tests and a complete blood count and platelet count may be worthwhile to avoid exacerbating an unrecognized underlying problem or to help interpret abnormal tests at a later date.

3. Routine blood and urine screening for severe anticonvulsant reactions is of no proven value and is not recommended in asymptomatic patients receiving antiepileptic medications.

4. Patients receiving anticonvulsant medication should contact their physician if they have a rash or unexplained illness. At that time, blood and urine tests could be considered.

5. Further research is needed to identify patients at risk for serious anticonvulsant reactions.

\section{Committee Members}

Peter Camfield

Carol Camfield

Joseph Dooley
Kevin Farrell

Pierre Langevin

Peter Humphreys

\section{REFERENCES}

1. DeVries SI. Haematological aspects during treatment with anticonvulsant drugs. Epilepsia 1965; 6:1-15.

2. Compendium of Pharmaceuticals and Specialties. Canadian Pharmaceutical Association 1988. Southam Murray. Toronto.

3. Physicians' Desk Reference, Medical Economics Co., Oradell. N.J., 1988.

4. Schmidt D. Adverse effects of antiepileptic drugs. New York: Raven Press, 1982.

5. Spielberg S, Gordon G, Blake D, Goldstein D, Herlong A. Predisposition to phenytoin hepatotoxicity assessed in vitro. $\mathrm{N}$ Engl J Med 1981; 305: 722-8.

6. Gerson W. Fine S, Speilberg S, Sensenbrenner L. Anticonvulsantinduced aplastic anemia: increased susceptibility to toxic drug metabolites in vitro. Blood 1983:61: 8, 889-893.

7. Fenichel GM. Valproate hepatotoxicity: two new cases, a summary of others and recommendations. Pediatric Neurology 1985: 1: 109-13.

8. Delattre JT, Safai B, Posner JB. Erythema multiforme and StevensJohnson Syndrome in patients receiving cranial irradiation and phenytoin. Neurology 1988: 38: 194-197.

9. Camfield C, Camfield P. Smith E, Tibbles JAR. Asymptomatic children with epilepsy: Little benefit from screening for anticonvulsant-induced liver, blood or renal damage. Neurology 1986; 36: 838-841.

10. Mattson RH, Cramer JA, Collins JF and the VA Epilepsy Cooperative Study Group. Connective tissue changes, hypersensitivity rash and blood laboratory test changes associated with antiepileptic drug therapy. Annals of Neurology 1986: 20: 119 . 120 (Abstract).

11. Silverstein FS, Boxer L, Johnstone M. Hematological monitoring during therapy with carbamazepine in children. Annals of Neurology 1983; 13: 685 (letter).

12. Willmore LJ, Wilder BJ, Bruni J. Villareal HJ. Effect of valproic acid on hepatic function. Neurology 1978: 28: 961-4.

13. Physicians' Desk Reference. Medical Economics Co., Oradell. N.J., 1988. p. 989.

14. Ibid. p. 511.

15. Dreifus FE, Santilli N, Langer DH, Sweeney KP, Moline KA, Menander KB. Valproic acid fatalities: A retrospective review. Neurology 1987; 37: 379-385.

\section{Screening of the Epileptic Patient - Is it worth the trouble?}

\author{
Robert H.A. Haslam and Gideon Koren \\ From the Department of Pediatrics and the Division of Clincial Pharmacology \& Toxicology. Hospital for Sick Children. Toronto, Ontario
}

Anyone who treats epileptic patients with anticonvulsants is wary of their potential side-effects which on rare occasion can cause death due to aplastic anemia, Stevens-Johnson syndrome or hepatotoxicity. Current dogma suggests that newly treated patients be followed with routine blood tests, including a complete blood count and liver function studies, particularly during the initial several months of treatment. It is the hope that an abnormal test will identify patients at risk for serious drug reactions and that promptly discontinuing the anticonvulsant will prevent the serious consequences. Unfortunately, such is not the case, as most deaths appear to be idiosyncratic and may occur in the face of relatively normal laboratory data. However, there are reports of patients who tolerate an anticonvulsant for months and then suddenly develop symptoms of toxicity. Some of these patients did not have routine blood screening nor symptoms of an idiosyncratic reaction prior to death.' Would screening have prevented fatal illness in such patients?

Camfield et al suggest that routine screening of asymptomatic patients is unwarranted as the exercise will not likely identify patients at risk and furthermore the costs would be prohibitive. They recommend that education of the parents or patient as to the early warning symptoms of an idiosyncratic reaction fol- 